\title{
Among Escherichia coli Genetic Stock Center strains many are resistant to cytotoxic antimetabolites
}

\author{
E. I. Cherepenko, O. I. Karpenko \\ Institute of Molecular Biology and Genetics, Ukrainian Academy of Sciences \\ Zabolotnoho st., 150, Kyiv, 03143, Ukraine
}

\begin{abstract}
Mutants resistant to cytotoxic antimetabolites arise in $E$. coli with a frequency about $10^{-4}$. Because most in this collection strains contacted a mutagen we checked how many strains could tolerate two inhibitors used: glyphosate and 6-aza-uracil. Among 28 strains studied 12 could tolerate these inhibitors.
\end{abstract}

One of the cytotoxic antimetabolites known as $(\mathrm{N}-$ [phosphonomethyl]-glycine is widely used as a herbicidal glyphosate. It is a target inhibitor inactivating the sixth enzyme of the shikimic pathway of the aromatic compound synthesis in plants, fungi and bacteria [1]. In studying the mechanisms of cytotoxic resistance to this antimetabolite in $E$. coli the following was observed. While screening the wild type cells on minimal M9 agar containing $0.5 \mathrm{mM}$ glyphosate no spontaneous glyphosate-resistant (gly $)$ mutants have been isolated indicating a gly mutation frequency less than $3 \cdot 10^{-8}$. However, after the treatment of these cells with nitrosoguanidine at both high and low cell viability an extraordinary large number of gly mutants arise at a frequency of about $1 \cdot 10^{-4}$. This is 2 orders of magnitude higher than the level of mutability of metabolic genes [2, 3]. Mapping of gly mutations showed that they are scattered around the entire chromosome [3]. Interestingly, half of the gly mutants emerged at both heavy and light mutagenesis has turned out to be multiple resistant. In our experiments they could tolerate not only glyphosate but also S-2-aminoethyl-L-cysteine («Sigma», USA) (AEC) and 6-aza-uracil (AU) (synthesized by Dr. Inna Alexeeva our Institute). The frequency of the $a^{r} c^{r}$ and $a u^{r}$ mutation arising was also about $1 \cdot 10^{-4}$ [3].

Since the majority of $E$. coli Genetic Stock Center strains have been mutagenized we were interested to inquire if and how often the mutations conferring resistance to cytotoxic antimetabolites could be found as nonselected mutations among these strains. For

(C) E. I. CHEREPENKO, o. I. KARPENKO, 2002 this study we have chosen 28 CGSC strains. They include $\mathrm{F}^{\prime}$ kit strains, the strains bearing markers in the $68-72 \mathrm{~min}$ section of $E$. coli chromosome and strains having mutations in genes encoding two aminoacyl-tRNA synthetases. The strains are listed in Table 1.

When tested to tolerate toxic amino acid and nucleic acid base analogues 10 strains of 28 studied appeared to tolerate either one of the inhibitors used or both of them as shown in Table 2. Since 5 of these

Table I

E. coli Genetic Stock Center strains used in this study

\begin{tabular}{l|l||l|l}
\hline \multicolumn{1}{c||}{ Designation } & CGSC & Designstion & CGS \\
\hline & & & \\
$\chi 53$ (OR 11) & 6350 & KL729 & 4260 \\
E5014 & 4288 & KL738 & 4289 \\
F500/GMS724 & 5505 & KL731 & 4254 \\
JE5550 & 5760 & KL732 & 4255 \\
KL701 & 4256 & KL723 & 4251 \\
KL703 & 4253 & TH 6 & 5670 \\
KL709 & 4279 & BSV 11 & 6991 \\
KL718 & 4287 & CAG18574 & 7439 \\
KL711 & 4291 & CAG12184 & 7437 \\
KL728 & 4258 & IQ 419 & 7132 \\
DFF1/JC1553 & 4326 & CAG12127 & 7441 \\
KL704 & 4280 & CAG12072 & 7440 \\
KL706 & 4265 & NP 37 & 4913 \\
KL708 & 4248 & HO 202 & 6340 \\
\hline
\end{tabular}


CHEREPENKO E. I. KARPENKO O. I.

Table 2

E. coli genetic stock center strains resistant to cytotoxic antimetabolites: glyphosate (GLY) and 6-azauracil (6-AU)

\begin{tabular}{|c|c|c|c|c|c|}
\hline \multirow[b]{2}{*}{ Strain } & \multirow[b]{2}{*}{ Sex } & \multirow[b]{2}{*}{ F', No } & \multirow[b]{2}{*}{ Chromosomal markers } & \multicolumn{2}{|c|}{ Resistance to: } \\
\hline & & & & $\begin{array}{c}\text { GLY } \\
(0,5 \text { mM })\end{array}$ & $\begin{array}{c}6-\mathrm{AU} \quad(200 \\
\mu \mathrm{g} / \mathrm{mI})\end{array}$ \\
\hline IQ419 & $\mathbf{F}^{-}$ & - & zha-2:Tn10, argG78, rpsL257 (str R) & + & + \\
\hline CAG12072 & $\mathbf{F}^{-}$ & - & $\lambda^{-}$, sfsB 203:Tn/0, rph-l & + & - \\
\hline BSV11 & $\mathbf{F}^{-}$ & - & $\begin{array}{l}\operatorname{gln} V 44(A S), \lambda^{-}, \operatorname{mcr} A, \operatorname{rfd} D I ?, \text { endAl, ribBIJ:TnI0, spoTI?, thi-1, mcrB, } \\
\text { hsdR29 }\end{array}$ & - & + \\
\hline E5014 & $F^{\prime}$ & 128 & $\Delta(g p t-l a c) 5, g \ln V 44(A S), \lambda^{-}$, relAI?, rpsE211(spcR), mal24, thi-I & + & + \\
\hline JE5550 & $\mathbf{F}^{\prime}$ & 506 & $\begin{array}{l}\operatorname{lac} Y, \operatorname{ts} x-9, \operatorname{gln} V 44(A S) ?, \operatorname{galK2}(O c), \operatorname{man} A 4, \operatorname{aroD} 6, \operatorname{gyr} A / 2(N a l R), \\
\operatorname{rec} A 1, \operatorname{rps} L 700(\operatorname{str} R), \operatorname{mlt}-1, \operatorname{argE} 3(O c)\end{array}$ & + & - \\
\hline DFF $1 / J C 1553$ & $F^{\prime}$ & 150 & $\begin{array}{l}\text { leuB6, fhuA2, lacYI, glnV44(AS), gal-6, } \lambda^{-}, \text {hisGI(Fs), recA } 1, \text { argG6, } \\
\text { rpsLI04, malTI }\left(\lambda^{\mathrm{r}}\right), \text { xylA7, mtlA2, metBI }\end{array}$ & + & + \\
\hline KL704 & $\mathbf{F}^{\prime}$ & 129 & as in DFF1/JC1553 & + & - \\
\hline KL728 & $F^{\prime}$ & 111 & as in DFF1/JCl 1553 & + & - \\
\hline KL729 & $\mathbf{F}^{\prime}$ & 112 & as in DFF1/JC1553 & + & - \\
\hline KL738 & $F^{\prime}$ & 140 & as in DFF1/JC1553 & + & - \\
\hline
\end{tabular}

strains, differing only in episome originate from the same source and all are gly ${ }^{\mathrm{r}}$ it may be concluded that all of them bear the same chromosomal mutation conferring gly ${ }^{r}$ resistance. As for $a u^{r}$ resistance only one of these strains (DFF1/JC1553) could tolerate this inhibitor, indicating that an episome present in this and not in other strains of this group might bear the mutation responsible for this resistance. The strain E5014 containing FB128 covering the region famous in experiments on adaptive mutations $[4,5]$ could also tolerate both inhibitors used. To find out whether this is an attribute of a chromosomal or episomal mutation we did routinely a conjugal transfer of this episome to a sensitive AB1157 strain, selecting pro $^{+}$exconjugants. They turned out to be sensitive to both inhibitors used, indicating that these mutations affect the chromosome in this strain.

Having studied 28 strains of CGSC collection taken rather randomly showed that 6 of them bore emerging at a high frequency mutations and conferring resistance to toxic antimetabolites. A preliminary test of the strains on their resistance to cytotoxic antimetabolites could be useful for some experiments.

о. Й. Черепенко, О. І. Карпенко

Багатьом штамам Escherichia coli з Генетичної колекції притаманна стійхість до цитотоксичних антиметаболітів

Резюме

Враховуючи, цо при мутагенному обробленні клітин кишкової палички частота виникнення мутантіе, стійких до цитотоксинних антиметаболітів, складас $I \cdot J^{-1}$, перевірено, як часто у цих итамах відбувасться зміна ознаки від цутливості до стійкості до двох інсібіторів: гліфосату та 6-аза-урацилу. 3 28 вивчених итамів 12 виявилися стійкими.

\section{Е. И. Черепенко, О. И. Карпенко}

Многие штаммы Escherichia coli из Генетической коллекции обладают устойчивостью к цитотоксическим антиметаболитам

Резюме

Учитывая, что при мутагенной обработке клеток кииечной палочки частота возникновения мутантов, устойчивьх к иитотоксическим антиметаболитам, составляет $1 \cdot 10^{-}$, проверено, как часто в этих итаммах происходиго изменение признака от чувствительности к устойчивости к двум ингибиторам: глифосату и 6-аза-урацилу. Нз 28 изученных итаммов 12 оказались устойчивыми.

\section{REFERENCES}

1. Kishore G., Shah D. Aminoacid biosynthesis inhibitors as herbicides // Ann. Rev. Biochem.-1988.-57.-P. 627-663.

2. Comai $L$, Sen $L$, Stalker $D$. An altered aro A gene product confers resistance to the herbicide glyphosate $/ /$ Science.1983.-221.-P. 370-371.

3. Cherepenko E. I., Karpenko O. I., Maliuta S. S. Genetic mechanisms of Escherichia coli resistance to aminoacid biosynthesis inhibitors: 1 . On genetic mechanisms of glyphosate resistance unrelated to the target gene // Biopolimery i kletka (Ukraine).-1994.-10.-P. 79-83.

4. Cairns J., Foster P. L. Adaptive reversion of a frameshift mutation in Escherichia coli // Genetics.-1991.-128.-P. $695-701$

5. Godoy V. G., Gisatullin $F$. S., Fox $M$. Some features of the mutability of bacteria during nonlethal selection // Genetics. $2000 .-154 .-$ P. 49-59

УДК $631.52+632.954 .576 .7$ Надійича до редакціі 28.12.01 